\title{
A CASE OF ADENO-CARCINOMA OF THE LOWER JAW, WITH PECULIAR METASTATIC DEPOSIT.
}

\author{
By EDMOND J. M'WEENEY, M.A., M.B.; \\ Pathologist, Mater Misericordiæ Hospital ; Lecturer on Pathology, Catholic \\ University School of Medicine.
}

[Read in the Pathological Section, Feb. 14th, 1890.]

TrE case, the details of which I have the honour of laying before the Academy this evening, seems to me worthy of notice on account of-

1st. The unusual occurrence of a tumour of such microscopic structure in connection with the lower jaw.

2nd. The peculiar situation of the only secondary deposit found, and

3rd. The divergence between the primary and the secondary growth in microsenpic structure, presenting a good example of carcinomatous change in the metastatic deposit from a purely adenomatous tumour.

CASE.-The patient, J. S., was a man, aged about seventy, admitted into the Mater Misericordiæ Hospital, October, 1889. The tumour sprang from the gum apparently by a broad base, just behind the lateral incisor on the right side. Here it formed a mass, about the size of a pigeon's egg, displacing the lateral incisor, which was carried altogether out of the alveolus and was supported on the tumour itself, to which it adhered by the gum, which was involved in the neoplasm. The consistency of the tumour was somewhat hard; it was covered with mucous membrane, and nowhere ulcerated. It had made its appearance about a year previously, and grown rapidly. The gums on each side of the tumour appeared thickened and infiltrated for a considerable area. Mr. Coppinger, under whose care the patient was, removed the 
front part of the lower jaw as far back on each side as it seemed affected-viz., as far as the first bicuspid; but no reaction was established, and the patient rapidly sank, dying twenty-four hours after the operation.

The post mortem notes are as follows :-

Autopsy twelve hours after death. Body emaciated; slight general icterus; rigor mortis extremely distinct. On opening the mouth the front of the lower jaw, as far back as the bicuspids on each side was wanting. No sign of reaction. Nodules somewhat similar in appearance to the tumour could be seen and felt beneath the tongue in the floor of the mouth.

Larynx and trachea, pharynx and cesophagus healthy; lungs, emphysematous; pleura generally adherent on right side.

Heart.-Slight mitral stenosis.

Abdomen.- The sigmoid flexure of the colon took a remarkable course. The descending colon having arrived at the brim of the pelvis, turned sharply upwards and inwards, rising to the level of the ensiform cartilage. It then turned downwards again, keeping to the right of the middle line, as far as the prominence of the sacrum, when it assumed a mesial position, and was continued as far as the rectum to the pelvic outlet. The part of the gut attached to it was enormously distended with gas, and occupied nearly the whole of the front of the abdominal cavity.

S'pleen.-Normal.

Kidneys.-Small, tough, fibroid.

Adrenals.-Normal.

Ureters and bladder.-Normal.

Liver.-Small, pale; very firmly fixed. On cutting into it in situ, considerable quantities of grumous brownish-yellow material exuded from orifices of bile ducts. Liver substance friable; colour pale. Gall-bladder very tense, dead white, matted together very closely with duodenum just outside pylorus, and hepatic flexure of colon, the whole constituting a dense white mass, the structures being cemented together by tough, evidently neoplastic, tissue. The walls of the gall-bladder were greatly thickened, and the contents were colourless and mucoid. The cystic duct was 
obliterated, ending blindly in the neoplasm, which was here very dense, creaking like scirrhus on being cut, and presenting numerous points of fatty degeneration. The liver itself was slightly invaded by the neoplasm in the neighbourhood of the portal fissure.

Common Bile Duct.-Wide enough to allow little finger to pass through it. It perforated the neoplasm and opened into the duodenum by means of an aperture nearly as large as a threepenny bit.

Pancreas.-Head increased in size, owing to invasion by the neoplasm; distinct boundary line between the tumour and the pancreatic tissue.

The lymphatics about coliac axis were infiltrated, and the vena cava and hepatic artery were also involved, but their lumina not encroached on.

Stomach and intestines healthy. The hepatic flexure of the colon and the commencement of the duodenum were fixed by the growth, but the mucous membrane was nowhere implicated.

On microscopic examination the tumour of the jaw and that in the pancreatic region were found to be similar in structure, with certain points of difference, which will be alluded to later on. They consisted of well-formed gland tubules which were lined with very long columnar epithelium and scattered through a connectivetissue stroma, studded with round and spindle-shaped nuclei.

Many of the tubules in the tumour of the jaw had their lumina filled up with material which looked like secretion. It was composed of structureless substance, thickly studded with small compound granules, and it quite distended some of the tubules.

A distinct basement membrane, with flattened nuclei was traceable, and the columnar cells were disposed on it with perfect regularity. The structure of the pancreatic tumour was somewhat similar; but the lumina of the tubules were much less distinct, and the connective tissue stroma more abundant.

As regards the relation of these two neoplastic masses to one another, the writer is disposed to look upon the jaw tumour as the primary, the other being merely a metastatic, deposit. It must be conceded that the occurrence of such a metastasis is unusual, 
and it may, perhaps, be well to give the reasons which inclined the writer to this view.

The jaw tumour is strictly regular in structure as regards the tubules. Nowhere is the basement membrane broken through by epithelial proliferation. The outline of the tubes is regular, close-packed as they are, and the lumen is distinct, whereas in the abdominal growth many of the tubules are converted into mere islets of epithelium, solid strands of the most irregular shape, ramifying through the stroma. In the neighbourhood of the pancreas the transition was beautifully gradual from the most typically glandular to the most atypical (carcinomatous) structure. It seems to me more probable that an adenomatous growth should give rise to (partly) carcinomatous metastases than that the reverse could be the case. Of course, the fact must not be overlooked that the bile ducts are very apt-much more so than the mucous membrane covering the gums (where the occurrence of normal gland tissue is doubtful) - to give origin to adenocarcinomatous growths. But a solitary mestastasis from bile ducts to the gums and floor of the mouth would be very unusual indeed-much more so than a mestastasis from mouth to bile ducts or liver. No trace of any tumour could be discovered in any part of the intestinal tract, though careful search was made, especially in the rectum. The case seems to me to possess several points of interest, which have induced me to bring it before the Pathological Section of the Academy of Medicine. 